\title{
APPLICATION OF SIMULATION MODELS IN CAPACITY CONSTRAINED RAIL CORRIDORS
}

\author{
Robert H. Leilich \\ Corporate Strategies, Inc. \\ 5415-A Backlick Road \\ Springfield, VA 22151, U.S.A.
}

\begin{abstract}
This paper focuses on the practical value of using and selecting rail simulation models to find real solutions to real problems in constrained traffic corridors. It discusses issues which simulation can address, where quantitative results cannot be derived by any other method. A second section highlights differences among rail simulation models and the suitability of each kind to different types of problems. The third section discusses some derived parametric line capacity relationships between physical plant characteristics and train operations.
\end{abstract}

\section{INTRODUCTION}

As little as 20 years ago, U.S. railroads were shedding excess capacity in an effort to eliminate under-used physical plant in order to improve asset utilization of "core" properties. Improvements in rail capital, labor, and fuel productivity, coupled with deregulation, has resulted in near unparalleled growth in rail traffic. Railroads are now faced with capacity issues of how to handle traffic growth, yet improve service reliability and, to a lesser extent, reduce transit times. On top of all this is increasing public and political interest in commuter rail, intercity passenger, and High Speed Rail service as a viable alternative to crowded highways and air congestion in high population corridors.

Without excess capacity to absorb traffic increases and new traffic and with the high cost and scarce capital to build new infrastructure, railroads and governments are turning more to simulation models to get more out of existing plant and equipment or find ways to increase plant capacity at the lowest cost.

\section{MODELING CONSTRAINED CORRIDORS -- SPECIFIC EXAMPLES}

\subsection{Raleigh-Durham-Charlotte Corridor}

A classic example illustrating line capacity issues is the Raleigh-Charlotte, NC corridor, especially a portion of the corridor between Raleigh and Durham. In this corridor, owned by the North Carolina Railroad, the Norfolk Southern (NS) Railroad normally operates six to eight trains a day plus additional trains during certain seasonal high traffic periods. CSX Transportation also operates more limited traffic between Raleigh and a point approximately 6 miles west, and in the Durham area.

Seemingly endless highway construction has not resolved traffic congestion as rapid population growth has kept up or exceeded highway investment. Plans are underway to provide a rail commuter service between Spring Forest (east of Raleigh) and Duke Medical Center (west of Durham). Amtrak and North Carolina DOT studies also indicate a significant traffic potential for High Speed Rail (HSR) and possibly more regular passenger service in the Federally designated Washington-RichmondRaleigh-Durham-Charlotte HSR corridor (which includes an abandoned CSXT main line between north of Raleigh and CSXT's main line at Petersburg,VA).

The proposed commuter service operator, Triangle Transit Authority, seeks to build a commuter line which provides reliable 15 minute headway service during rush hour and 30 minute schedules in non-rush hours. A single track railroad with precisely located passing sidings is desired because capital limitations prevent building a double track line at this time. Affecting the location and length of passing sidings are tightly related interdependencies between location of stations, equipment behavior characteristics, maximum operating speeds, vertical and horizontal curvatures, station dwell times, and departure times from each end of the line.

Because some passing sidings might be located in areas of high expense (such as on long bridges or areas of 
closely spaced, high density road crossings) the length and location of sidings may have to be compromised with unknown affects on schedule performance. The choice of high or low level station platforms will affect station dwell times. The addition of the new station may upset the critical balance of the plant designed for the prescribed operating schedule. Unknown is the degree to which late trains can be tolerated, with subsequent questions of:

- When should a schedule be annulled;

- Should a late train be joined with a following onschedule train; or,

- Should all trains drop back to the schedule of the latest train to maintain operating symmetry?

In view of a long range plan to build a double track operations, another major consideration is whether certain physically constrained locations on the designed route can be permanently limited to one track. If so, for what distance can a single track be tolerated without affecting merits and benefits of an otherwise double track rail operation? Finally, both NS and CSXT want to preserve all options to increase freight services in the corridor as a result of the Conrail merger and continuing traffic growth.

Under the most inflexible alternative, each operation would be on tracks dedicated to each operation (commuter, High Speed Rail and regular passenger, and freight), each having its own passing tracks. Preliminary studies in this corridor have raised the following issues:

- The railroads will not give up line capacity which affects service reliability, flexibility, and ability to handle future traffic;

- Though there are some exceptions, railroads generally feel lighter weight commuter equipment, High Speed trains, and freight trains do not make good bed mates;

- Physical constraints exist within the corridor which inhibit building additional tracks;

- Capital cost of eliminating all constraints could make commuter rail and/or the High Speed Rail potential economically unfeasible;

- Providing commuter rail in the constrained corridor may limit options available to High Speed Rail or vice versa unless a joint, coordinated development plan is prepared;

- Building commuter rail facilities to preserve all options for High Speed Rail could increase construction cost and delays as cost sharing issues are resolved and funding is secured.

What is largely a single track corridor, could conceivably become a four track corridor between Raleigh and Durham plus a fifth main track for HSR passenger train meets. Proposed, but not necessarily feasible or acceptable, solutions to the above include:

- Shared use of freight railroad tracks with either HSR or commuter rail in constrained areas;

- Forgoing the option of a double track commuter rail line in constrained areas;

- Operating HSR passenger trains at limited speeds on the freight railroads in critical locations.

An alternative worth considering under the shared use concept is the preferred use of a dedicated line for HSR trains and the use of the NS main line as a flexible passing track. Conversely, the essentially single track NS mainline could utilize the HSR main line as a flexible passing track for meeting and passing other freight trains. It could relieve both operators of the cost of building dedicated long passing sidings, especially in constrained locations. Each other's track also could provide a bypass track for increasing time blocks for maintenance of way activities.

There are so many combinations of alternatives and many uncertainties with future traffic schedules and volume that traditional approaches to traffic planning (which provide excess capacity to accommodate contingencies) are unfeasible. Project capital costs are so high that no responsible party will make decisions without convincing evidence acceptable solutions.

Other questions to be resolved include:

- How do track maintenance windows affect service, and service reliability (both on the affected track and adjacent track where slow orders may be imposed for safety)?

- Are there trade offs where railroads may accept some additional traffic delays in certain constrained corridors in return for offsetting relief in other areas (a quid pro quo solution)?

- How much flexibility and service reliability is lost with each alternative solution?

- What are the service benefit/cost trade offs among different alternatives?

- Which compromise solution(s) is (are) fairest to all parties?

\subsection{Memphis Terminal}

A second example illustrates rail operations in the Memphis Terminal area. Five freight railroads are experiencing increased traffic congestion and delay, attributable to traffic growth. The Port of Memphis wants to build a new intermodal terminal, called the Super Terminal, encouraging railroads to concentrate the handling of all or most of their intermodal traffic at this facility. While the concept makes great economic sense, a 


\section{Application of Simulation Models in Capacity Constrained Rail Corridors}

major concern is the need to expedite movements of trains into and out of the terminal.

There are many options of physical plant improvements and train control issues which need to be resolved in order to make the whole terminal area function more smoothly. Even though alternatives can be identified, their probable service and cost impacts of on operations could not be determined without simulation. Simulation showed that the proposed Super Terminal, along with physical plant and train control improvements could dramatically reduce rail traffic congestion and operating costs and increase traffic capacity and service reliability.

Since terminal improvements will radically modify the way traffic is handled, four fundamental questions needed to be answered by the simulation:

- Will it work as intended?

- What is the best engineering and operating plan?

- Is it cost-effective?, and

- How are the benefits distributed?

\subsection{New Amtrak Service}

Privately owned businesses and the city of Las Vegas want Amtrak to run a privately financed fast passenger train between Los Angeles and Las Vegas. A portion of the operation would run over Union Pacific's heavily trafficked main line between Daggett, CA and Las Vegas, NV. The line is mostly single track, with portions of double track and many sidings that are shorter than the length of some trains that operate over the line. The UP needed to determine what changes in physical plant are necessary to offset or avoid delays that the proposed passenger service may inflict on freight operations.

Because operations vary greatly from day to day, simulations for a single seven day period were deemed not sufficient to evaluate the impact of different alternatives. It was important in all simulations that normal randomized variability of operations be simulated over a sufficient period of time that some statistical confidence can be derived by simulation results. In this case, UP analysts felt that the analysis of the Base Case and alternatives required the running of approximately 180 simulations, totaling 1,300 days of operation and the dispatch of 60,000 trains. Fifty megabytes of simulation output were processed by Paradox and Access data base management software to make statistical comparisons among the many simulations. Results provided some level of confidence in quantified operating differences among alternatives.

Without simulations, the impact of additional delays on traffic could not be quantified or the offsetting impact of physical plant modifications. Simulation provided quantitative results of alternatives - focal points for negotiations between the two parties.

\subsection{Other Examples}

The number of railioads and communities facing situations similar to the above examples is growing. A few of many examples include the Vancouver, British Columbia area; Passenger, HSR, and Commuter services in the Oceanside to San Diego, CA corridor; Commuter, Passenger, and Freight services in the Miami County, FL corridor; and rail traffic congestion in many major terminal areas, most dramatically illustrated by problems experienced by the Union Pacific.

\section{SIMULATION MODEL ALTERNATIVES}

Clearly, the only way to resolve thorny issues raised in the above examples is through the simulation of operations. Simulations must be credible, reliable, accurate, and acceptable by all parties, without which, no consensus for compromise solution can be reached. Modeling of rail operations is increasingly popular as their capability, ease of use, and credibility improves.

A decision to use a rail operations simulation model is summarized in Figure 1. Properly done, rail operations modeling is a consensus builder to designing a more modern transportation system serving all interests.

Key to any rail operations simulation is:

- Defining simulation study objectives;

- Obtaining accurate and complete data;

- Calibrating Base Case operations with real world operations, if possible;

- Achieving consensus on the Base Case (existing operations, if they exist);

- Identifying alternatives to be evaluated; and,

- Converting performance findings to measures of service impact, capacity, and economics.

There are four basic types of rail operations simulation models.

\subsection{Route Seeking Models}

Route seeking models are based on the concept of threading trains through vacant time/segment slots over a route or network. Trains, in order of highest priority are first routed over the line or network of lines in the system. Lower priority trains must operate over segments or tracks which are not occupied at the same time by higher priority trains. This form of modeling works reasonably well in environments where there are few differences in train priority, severe capacity limiting constraints do not exist, 


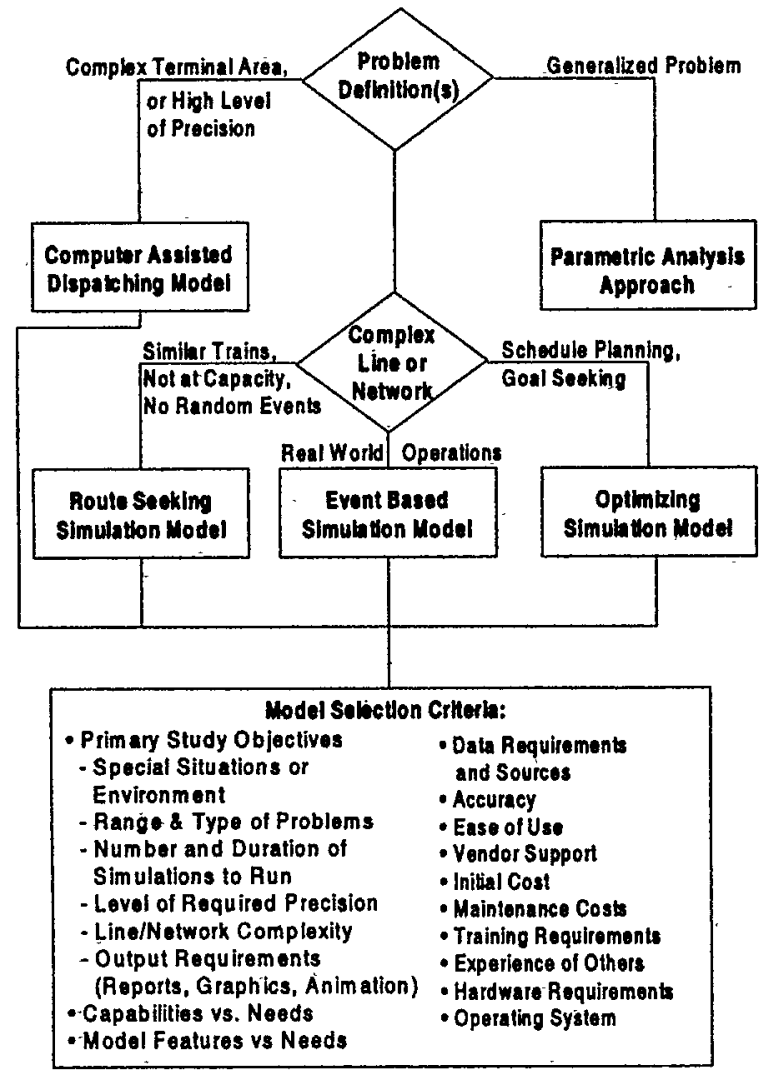

Figure 1: Using a Rail Operations Simulation Model

special events are limited to well defined delays, there are no conditional interdependencies among trains (especially those of different priorities), and there are no random delay events among low priority trains (which could affect high priority trains which have already been "dispatched").

Route seeking models are not true dispatch simulation models where trains of differing priorities interact with one another throughout a simulation. Route seeking models do not work well where random events frequently occur, train interdependencies exist, or the line or network is near capacity. These models require considerable time and effort to set up and run.

\subsection{Optimization Models}

Optimization models may or may not be event driven (see description following), but they rely on complex mathematical algorithms to systematically develop an operating plan that maximizes operating performance. While such models are good at squeezing maximum performance out of $a_{s}$ line or network, they tend to have limited capabilities with respect to handling Special Events, interdependencies among trains, and random events. These models can also be time consuming to set up and run. By their very nature they tend to out-perform what train dispatchers can do in a real world environment.

\subsection{Computer Assisted Dispatching Models}

These models require the user to prescribe routing movements and make many dispatch decisions. The real strength of these models is their ability to accurately map train movements. Their ability to monitor and display the movement of trains through complex interlockings where multiple paths exist cannot be easily duplicated by any other type of model. These models require significant user interaction when either the model cannot resolve a conflict. These models are among the most time consuming and laborious to code and run.

\subsection{Event Based Simulation Models}

True Event Based simulation models make dispatching decision comparable to that of good dispatchers. The best of these models can handle virtually every type of Special Event which, singularly or in combination, can replicate the occurrence of virtually any event experienced in railroad operations. Although some optimizing logic may be built into decision making, the optimizing algorithms are not as rigorous as those in Optimization models. Generally, Event Based models come closest to reproducing real world performance. There is a significant range of performance among models in this category. Some are difficult to code and operate and may fail in highly complex operating environments (requiring manual intervention to resolve a train conflict).

A valuable feature in some true event based simulation models is the ability to inject random dispatches and events which reflect real world occurrences. By running a simulation over a period of two weeks (or even more), improved measures of system performance, or measures of capacity, can be obtained.

\subsection{Model Selection}

The ability of the four model types to duplicate performance on a micro (mile-by-mile) basis versus a macro (point-to-point) basis varies significantly. Link and node based models usually perform best on a micro level. The ability of Event Based models to handle high volumes of trains over long distances and many days of simulation also varies significantly.

The greatest confidence in a model is gained when it can be calibrated to known, quantified, historical behavior over a line or network and alternatives to be evaluated do not wander far from the Base Case. In situations where whole new services are being prescribed or new lines are being built, the historical ability of the model to perform accurately and reliably in a wide range of environments 
must be relied upon as the basis for accepting simulation results.

Some simulation models have built in Train Performance Calculators (TPC). Most rely on using unrestricted segment running times developed by an external TPC. Most models make adjustments for unrestricted segment running times based on the occurrence of delays which a train may experience. It is essential that the TPC used duplicates actual train performance, as segment running times significantly affect simulation results.

Simulation models which rely on externally developed TPC unrestricted running times typically use empirical formulas to accelerate and decelerate trains within the simulation model. This approach may not take into account the effects of grades, under the assumption that differences in deceleration are offset by differences in acceleration. Also, signal block spacings in some models are based on running times and not actual location. Consequently, the location of trains between stations (control points) varies in precision among the models. There is often a classic trade off between the time and cost of setting up a model versus level of precision in the results. In short, the model to select for the simulation of rail operations is a factor of many things.

\section{USE OF SIMULATION TO DEVELOP PARAMETRIC RELATIONSHIPS}

In the late 1970's, under a Federal Railroad Administration (FRA) contract, Peat, Marwick \& Mitchell, Co. (PMM\&Co) examined statistical relationships between line capacity, as measured by total train delays, and factors which most likely affect line capacity. Relationships were presumed to exist among:

- Types of trains operated (as measured by powerto-weight ratios);

- Number, length, and frequency of passing sidings;

- Type of signal system;

- Average train speed; and,

- Traffic peaking factors.

Findings from this study represented the most comprehensive analysis of the most important factors that affect line capacity. One graphic from this study is shown in Figure 2.

Results from the study led to the development of a Parametric Model of Line Capacity, programmed to operate on an Apple II computer.

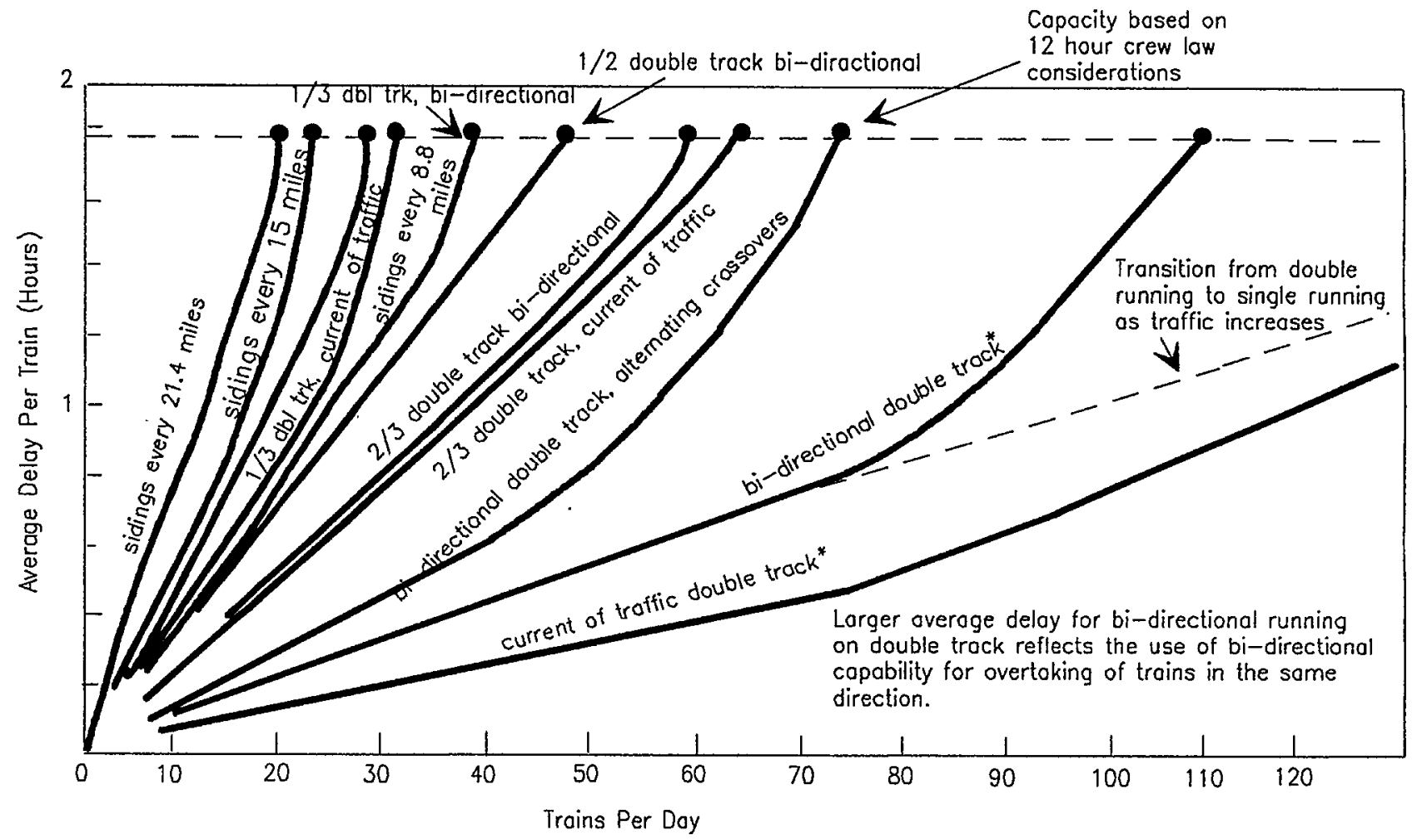

Figure 2: Train Volume - Average Delay Relationships for Alternative Configurations of 100 Mile Rail Line 


\subsection{Track Maintenance Window}

A concern to both operating and engineering departments on lines with frequent train operations are trade offs between providing maintenance time windows and delaying trains. The PMM\&Co study defined the time required for a train to pass a work area of known length to be:

$$
T_{p}=\frac{\left(.568 C+60 D_{s}\right)}{V_{s}}
$$

where:

$T_{p}=$ time to pass work area, in minutes

$\mathrm{C}=$ number of $50 \mathrm{ft}(15 \mathrm{~m})$ equivalent cars in train

$\mathrm{D}_{\mathrm{s}}=$ length of work area (in miles, or $\mathrm{Km}$ )

$\mathrm{V}_{\mathrm{s}}=$ slow order train speed in MPH or KPH

.568 and $60=$ conversion factors (English or Metric)

This formula, however, reflects only a portion of maintenance-of-way downtime. Influences of train fleeting

$$
\text { Total } M / W \text { delay }=\sum_{n=I}^{f}\left\{T_{c}+\sum_{p=1}^{N}\left[T_{p}+s\right]+T_{v}\right\}
$$

and other considerations are included in the formula:

where:

$\mathrm{T}_{\mathrm{c}}=$ clearance time (minutes in advance of arriving train, beginning with time when productive on track work must stop)

$T_{p}=$ train passing time (determined in previous formula)

$\mathrm{T}_{\mathrm{v}}=$ setup time required after train passage (minutes)

$\mathrm{N}=$ number of trains in fleet passing work site

$\mathrm{S}=$ time interval to next train in fleet (minutes)

$f=$ number of train fleets per workday

It is important to note that when trains are not fleeted, but instead arrive individually, each train becomes a "fleet" with $S=0$. The following example illustrates the use of formulas. Table llists the trains used in the example.
Table 1: Inputs to $M / W$ Train Delay Formula

\begin{tabular}{|c|c|c|c|}
\hline Train & No. of Cars & Direction & Arr. Time \\
\hline 1 & 50 & East & 8:30 AM \\
\hline 2 & 125 & West & $11: 00 \mathrm{AM}$ \\
\hline 3 & 75 & East & $1: 10 \mathrm{PM}$ \\
\hline 4 & 60 & East & $3: 20 \mathrm{PM}$ \\
\hline 5 & 100 & West & $3: 50 \mathrm{PM}$ \\
\hline
\end{tabular}

1. A certain maintenance operation requires a start-up time $\left(T_{v}\right)$ of 15 minutes;

2. Ten minutes is required to clear equipment in advance of a train's arrival;

3. Minimum productive track time is one hour;

4. Work is on a 2-mile stretch of single track; and,

5. All trains must pass the work site at $15 \mathrm{MPH}$.

$\mathbf{T}_{\mathbf{p}}$ train passing times are computed as follows:

$T_{1}=9.89$ minutes $T_{4}=10.27$ minutes

$T_{2}=12.73$ minutes $T_{5}=11.79$ minutes

$\mathrm{T}_{3}=10.84$ minutes

Trains 4 and 5 pass the work site within an hour of each other, and thus are treated as a "fleet." The time $\mathbf{S}$ between the clearance of train 4 and the arrival of train 5 is 19.73 minutes $(3: 50-3: 20-10.27)$. The three other trains are, in effect, "fleets" of one train each. Thus, total delay resulting from the train passage equals:

$$
\begin{aligned}
& \text { Delay }_{2}=(10+12.73+15) \quad=37.73 \\
& \text { Delay }_{3}=(10+10.84+15) \quad=35.48 \\
& \text { Delay }_{4}=(10+10.27+19.73+11.79+15)=\underline{66.79} \\
& \text { Total Delay } \\
& 175.25
\end{aligned}
$$

The example illustrates that even a relatively light volume of traffic on a single-track line can cause almost three hours down time for the $\mathrm{M} / \mathrm{W}$ crew out of an eight hour work day - a 38 percent reduction in available time. Additionally, the remaining work "slots" are only slightly above the minimum on-track requirement for work.

Complementing the use of parametric relationships, stringline or computer printed outputs from a simulation model may be used to evaluate $M / W$ requirements at selected points and to test policy alternatives of holding or rescheduling trains to increase $\mathrm{M} / \mathrm{W}$ productivity. The model can be used to estimate "recovery" - the time to absorb traffic delays and permit trains to resume a normal operating schedule. 


\section{Application of Simulation Models in Capacity Constrained Rail Corridors}

\subsection{Line Capacity Monitoring System}

In mid-1995, the Canadian National wanted a much faster tool than a simulation model to evaluate operating performance as measured against "working" and theoretical line capacities. With the aid of Corporate Strategies, Inc. (CSI), source code for the original parametric model was converted to a PC-based Windows program. The $\mathrm{CN}$ made major enhancements to the converted model. Its objective was to monitor operating performance on key subdivisions on a daily, weekly, monthly, and annual basis. The improved model will useful in helping the company run a more scheduled railroad and providing early warning for lines nearing capacity.

Using CSI's Railway Analysis and Interactive Line Simulator (RAIISTM) model, hundreds of simulations were run to define statistical relationships between physical plant and train characteristics, signalization, and operating practices. While details of CN's research are not public, it concluded:

- Generally linear or upward relationships exist between the number of trains per day and average delay per train. Consequently a squared or stronger relationship exists between number of trains and total delay.

- The relationship among line and operating characteristics is additive rather that multiplicative. Consequently improving one aspect while ignoring other ultimately results in diminishing returns.

- Most economically-operated lines reach capacity based on service level long before absolute or jam capacity is reached.

\subsubsection{Approach to Capacity Estimation}

Each line segment has a delay (slope) characteristic, $\mathbf{K}$, measured in hours per 100 miles, based on its particular parameters of plant and operation. Capacity, $\mathbf{C}$, of a line, as measured in trains per day, is related to its delay characteristic $\mathbf{D}$ based on the maximum average delay for trains, $\mathbf{A}_{\mathbf{c}}$, as follows:

$$
C=\frac{A_{C}}{K}\left(\frac{100}{L}\right)
$$

where $\mathbf{L}$ is the length of the line segment in miles.

$\mathbf{A}_{\mathbf{c}}$ is the average delay per train (in hours, exclusive of schedule delay), which itself is a function of:

- $\quad$ M - Maximum acceptable trip time for critical trains

- $\quad S$ - Speed of the slowest class of trains
- $\quad$ P - a traffic peaking factor

- $\quad$ D - a directional imbalance factor

- I - intermediate required delays, such as work by critical trains

Critical trains are not necessarily the fastest or highest priority. Critical trains are the first which may exceed maximum acceptable trip time. For example, if high priority trains still traverse a line in acceptable times, but low priority trains are exceeding hours of service rules (possibly because of both en route delays and intermediate work), then the low priority trains are the critical trains.

Parametric factors included in the analysis of comparing a particular level of delay characteristic, $K$, to a reference delay case, $\mathbf{K}_{\mathbf{0}}$ is a linear relationship:

$$
\mathrm{K}=\mathrm{f}_{\mathrm{m}} \mathrm{K}_{0}
$$

where $\mathbf{f}_{\mathbf{m}}$ is the additive relationship among the factors which increase or decrease capacity:

$$
\left.f m=\left[\left(\sum f_{0 i}^{P i}\right)-\left(N_{I}-1\right)\right]\left(\sum_{f_{0 i}}^{-P i}\right)-\left(N_{D}-1\right)\right]^{-1}
$$

The first bracketed term represents capacity factors which, as they increase, also increase capacity. The second bracketed term represents capacity factors which decrease capacity as the values increase. The $f_{0 i}$ 's are the coefficients for each individual factor. The $P_{1}$ 's are the proportional change in the factor, and the $N_{\mathbb{I}}$ and $N_{D}$ are the number of increasing and decreasing capacity factors respectively.

The proportional change in a factor from the reference base is normalized to an average $\left(\mathbf{P}_{\mathrm{i}}\right)$, so the change will work continuously whether increasing or decreasing:

$$
P_{i}=\frac{\left(V_{i}-V_{0}\right)}{1 / 2\left(V_{i}+V_{0}\right)}
$$

where $V_{i}$ is the new value of the traffic peaking $P$ parameter and $V_{0}$ is the base or original value of the $P$ parameter. 


\subsubsection{Factors Included in the Parametric Model}

Several factors are included directly in the determination of capacity as shown in equation (1) - peaking, directional imbalances, a measure of speed, and intermediate work requirements. Most other factors are brought in through modifications of the $K$ value in equations (2), (3), and (4). Below are the factors included in the parametric model and the range of sensitivities associated with the factors. Note that $\mathbf{f}_{\mathrm{oi}}$ 's which are greater than 1 increase the $\mathbf{K}$ value, hence increase delays to trains as the factors they represent increase. $f_{0 i}$ 's which are less than 1 decrease delays as the factors increase. The further the value is from 1 , the more sensitive delays and hence capacity are to changes in the respective factors. The ranges for some factors are an indication of very non-linear relationships.

Table 2: Parametric Model Input Factors

\begin{tabular}{lcc}
$\begin{array}{l}\text { Capacity Factor } \\
\text { Siding or }\end{array}$ & $\begin{array}{c}\text { Single } \\
\text { Track } \mathrm{f}_{\text {oi }}\end{array}$ & $\begin{array}{c}\text { Double } \\
\text { Track }\end{array}$ \\
$\begin{array}{l}\text { Crossover } \\
\text { Spacing }\end{array}$ & 1.78 to 2.86 & 1.48 \\
$\begin{array}{l}\text { Uniformity or } \\
\text { Siding Spacing }\end{array}$ & 0.79 & N/A \\
$\begin{array}{l}\text { Block Length } \\
\text { Average Running }\end{array}$ & 1.15 to 1.54 & 1.57 to 2.10 \\
Speed & 0.11 to 0.48 & 0.13 to 0.33 \\
$\begin{array}{l}\text { Uniformity of } \\
\text { Speed }\end{array}$ & 0.71 & N/A \\
$\begin{array}{l}\text { Shifting Peaking } \\
\begin{array}{l}\text { Direction } \\
\text { Imbalances }\end{array}\end{array}$ & 0.69 & 0.67 \\
$\begin{array}{l}\text { Reducing } \\
\text { Unplanned } \\
\text { Delays }\end{array}$ & 0.75 & 0.97 \\
$\begin{array}{l}\text { Reducing } \\
\text { Priorities }\end{array}$ & 0.82 & 0.72 \\
$\begin{array}{l}\text { Partial Double } \\
\text { Track }\end{array}$ & 0.67 to 0.95 & N/A \\
\hline
\end{tabular}

\subsubsection{Implications of Factors}

Values based on simulations were compared to actual rail lines for validation, and affirmed that train delay sensitivities and parametric model results were consistent with actual experience.

Some implications can be drawn from the above numbers, although they do not directly indicate what are the best actions to take to increase capacity, since some are obviously much more difficult to implement than others.

Siding Spacing vs. Crossover Spacing: Proportionately at least, siding spacing is more important on single track than crossover spacing is on double track. Capacity is less sensitive as average siding or crossover spacing decreases.

Signal Spacing: Signal spacing is significantly more important on double track than it is on single track.

Average Running Speed: Average speed is very important to capacity on both single and double track perhaps the single most important factor under most circumstances. The sensitivity is greatest at slowest speeds and above $50 \mathrm{mph}$ has considerably less effect.

Proportion of Double Track: The portion of double track on a line has a significant, but moderate effect on capacity. Sensitivity is greatest when a line is mostly double track, i.e., even small sections of single track can be fairly disruptive of line capacity.

Other Factors: Peaking, directional imbalances, unplanned delays, and priorities all have significant, but more moderate effects on capacity.

\subsubsection{Specific Inputs to the Current Parametric Capacity Model}

The specific inputs to the current model are:

- Description of line segment(s) to be analyzed;

- Miles of single track and miles of double track main line under ABS (Absolute Block Signal);

- $\quad$ Miles of single track and miles of double track under CTC (Centralized Traffic Control);

- Miles and number of other signaled tracks (to allow for short sections of three or more tracks when traffic and capacity are not uniform);

- Number of intermediate stations plus one (stations for the parametric model include operating or controlled sidings on single track where trains usually meet, significant crossovers on multiple track sections, and points where single track becomes double track or vice versa);

- Number of intermediate yards (used only in large studies where minimal operating information is available);

- Maximum speed (generally maximum average speed for the fastest trains on the line segment. If only one passenger train a day operates but a number of fast intermodal trains, the intermodal train limit would be most appropriate); 


\section{Application of Simulation Models in Capacity Constrained Rail Corridors}

- Speed ratio (fastest/slowest) (While not documented in the early PPM \& Co. model it represents the ratio of the average free-running speed of the fastest group of trains to the average of the slowest group);

- Grade adjustment (to account for trains being less on steeper grades) trains are able to operate speed at limits. The values are:

\begin{tabular}{llc} 
Grade & Ruling & Amount Added to \\
Severity & Gradient & Min. Running Time \\
\cline { 2 - 3 } N (Normal) & Up to 1\% & $10 \%$ \\
H (Heavy) Up to 2\% & $15 \%$ \\
M (Mountain) Over 2\% & $20 \%$
\end{tabular}

- Speed restrictions and lengths of restrictions (used in conjunction with maximum speed to estimate minimum running times over a line);

- Stop time (an allowance, in hours, for planned en route delays for critical trains in addition to yard delays);

- Average signal block length (miles);

- Segment uniformity (measure of the uniformity of spacing between control points) The default case has a average spacing of 8.82 miles with a variance of 3.87 miles (statistically, one standard deviation) or 0.44 of the average segment length:

\begin{tabular}{lc} 
Type of Uniformity & Variance \\
\cline { 1 - 2 } U (Uniform) & 0.00 \\
S (Semiuniform) & 0.22 \\
N (Nonuniform) & 0.44
\end{tabular}

- Maximum permitted trip time (the program can estimate the maximum hours allowable for critical trains, or it can be directly specified). The program estimates the time as the shortest of the following:

- (Line Length)/12.5 - 1

- (Line Length)/(45 percent of average speed)

- (Line Length)/18

- Siding Capacity (feet) of shortest siding usable by all trains (Controls car throughput on single track lines);

- Percentage of High Priority Trains (number of highest priority group of trains relative to total trains); and,

- Incidents (the level of unplanned incidents which cause train delays on a line - signal failures, hot boxes, dragging equipment, etc.) Levels are High, Low, and None.

\section{CONCLUSIONS}

While railroad operations simulation is now a proven analysis tool and increasingly used by railroads around the world, all models, by some standards, are still relatively primitive. All of the most popular simulation models have their roots in addressing very specific problems. Each has progressed on a shoe-string budget or as a labor of love by its developer(s). Some models are impressive and sexy in their appearance, but weak in their logic and performance. Others have better logic but are hard to use. All of them have limitations. For most applications, models should not make decisions that are better than state-of-the-art in real world dispatch decision making.

The ultimate benefit of modeling railroad operations is first, to give management a better understanding of operating relationships which affect service performance, cost and capacity; and second, to help management make better informed and cost effective decisions with respect to plant or other modifications which improve service performance and reliability. The airline industry has spent tens of millions of dollars to find good asset utilization solutions to the classic plane scheduling problem. The railroad industry has spent only a fraction of that amount to develop comparable tools to improve performance of its more than $\$ 70$ billion in plant and equipment assets.

Operations modeling has a definite place in improving railroad productivity, providing more competitive services, and increasing capacity to handle a wider variety of services.

\section{REFERENCES}

Krueger, H. 1997. Parametric Analysis Line Capacity Model. Unpublished Canadian National paper.

Leilich, R.H. 1994. Parametric Factors Affecting Rail Line Capacity. Prepared for World Bank, Washington, D.C.

Prokopy, J. C. and R.B. Rubin. 1975. Parametric Analysis of Railway Line Capacity. Federal Railroad Administration Report No. DOT-FR-5014-2.

\section{AUTHOR BIOGRAPHY}

ROBERT H. LEILICH is President of Corporate Strategies, Inc. (CSI), specializing in railway operations modeling and strategic planning. He has railway operating management experience with the former Santa $\mathrm{Fe}$ and consulting experience with A.T. Kearney, Inc. and Peat, Marwick, Mitchell \& Co. He started CSI in 1980. He has a Graduate Certificate in Transportation Economics from Yale University, a Masters degree in Industrial Management and a B.S. degree in Mechanical Engineering from Purdue University. $\mathrm{He}$ is a committee member with the Transportation Research Board, and a member of the American Society of Mechanical Engineers and INFORMS. He is a certified Surface Transportation Board Practitioner. 